\title{
INTERVENÇÕES EDUCATIVAS E SEU IMPACTO NA CULTURA DE SEGURANÇA: UMA REVISÃO INTEGRATIVA
}

Monique Mendes Marinho1, Vera Radïnz², Francis Solange Vieira Tourinho², Luciana Martins da Rosa², Marciele Misiak³

Objetivo: analisar as produções científicas sobre intervenções educativas desenvolvidas com profissionais de enfermagem voltadas para melhorar a segurança do paciente e que avaliaram a cultura de segurança após essa intervenção. Metodologia: utilizou-se a revisão integrativa de literatura eletrônica em seis bases de dados, com descritores específicos para cada base, publicados no período de janeiro de 2009 a novembro de 2014. Resultados: foram submetidos à análise nove estudos, resultando em duas categorias: Treinamentos didáticos e Programas Especificos de Segurança do Paciente. Conclusão: as produções apontaram atividades educativas que podem incentivar e melhorar a cultura de segurança do paciente.

Descritores: Segurança do Paciente; Cultura; Educação; Capacitação em Serviço; Enfermagem.

\section{EDUCATIONAL INTERVENTION AND IMPACT ON SAFETY CULTURE: AN INTEGRATIVE REVIEW}

Objective: to know the scientific productions about educational interventions developed with nursing professionals aimed at improving patient safety and that evaluated the safety culture after this intervention. Methodology: it was the integrative review of electronic literature search in six data bases with specific descriptors for each base, published between January 2009 and November 2014. Results: nine studies were submitted to analysis, resulting in two categories: Didactic Training and Specific Programs of Patient Safety. Conclusion: the productions aimed at educational activities that can stimulate and improve the culture of patient safety.

Descriptors: Patient Safety; Culture; Education; Inservice Training; Nursing.

\section{INTERVENCIÓN EDUCATIVA Y SU IMPACTO EN LA CULTURA DE SEGURIDAD: UNA REVISIÓN INTEGRADORA}

Objetivo: conocer las producciones científicas sobre intervenciones educativas desarrolladas con profesionales de enfermería orientadas a mejorar la seguridad del paciente que evaluaron la cultura de la seguridad después de esta interveción. Metodologia: se ha utilizado la revisión integradora de literatura electrónica en seis bancos de datos con descriptores específicos para cada banco, publicados en el periodo de enero de 2009 a noviembre del 2014. Resultados: fueron submetidas al análisis nueve estudios, resultando en dos categorias: Entrenamientos didacticos y Programas Específicos de Seguridad del Paciente. Conclusion: las producciones apuntaron actividades educativas que pueden incentivar y mejorar la cultura de seguridad del paciente.

Descriptores: Seguridad del Paciente; Cultura; Educación; Capacitación en Servicio Enfermería.

${ }^{1}$ Enfermeira. Doutoranda em Enfermagem. Universidade Federal de Santa Catarina (UFSC). E mail: moniquemarinho@yahoo.com.br ${ }^{2} 2$ Enfermeira. Doutora em Enfermagem. Docente de Enfermagem da UFSC.

${ }^{3}$ Enfermeira. Doutoranda em Enfermagem. UFSC. 


\section{INTRODUÇÃO}

A segurança do paciente tornou-se uma área prioritária, internacionalmente, devido à persistência de danos aos pacientes, com altos custos para os serviços de saúde, e comprometimento psicológico e físico dos pacientes e profissionais ${ }^{(1)}$. Diariamente, milhares de erros ocorrem na área da saúde, causando mortes e lesões incapacitantes em vários pacientes, tornando uma fonte importante de morbidade e mortalidade mundialmente ${ }^{(2)}$.

Diante do cenário de preocupação com a segurança, pesquisadores têm desenvolvido estudos para identificar ações que possibilitem a redução de danos e que promovam melhorias no atendimento dos pacientes, com mudança na cultura das organizações e dos profissionais ${ }^{(2)}$.

Muitas ações desenvolvidas para melhorar a segurança do paciente são atividades educativas aos profissionais de saúde, no próprio local de trabalho, proporcionando melhorias na cultura. As mudanças positivas na cultura permitem que a força de trabalho e os processos estejam voltados à melhoria da confiança e segurança dos cuidados, enfraquecendo a cultura de que o erro seja atribuido à incompetência da ação humana(3).

Estratégias para promover uma cultura de segurança pode incluir uma intervenção única ou intervenções combinadas, como o treinamento da equipe, que utiliza métodos estruturados para otimização dos processos de trabalho em equipe, como comunicação, cooperação, habilidades, atitudes e liderança ${ }^{(4)}$. Essas estratégias escolhidas e aplicadas por uma instituição de saúde como ferramentas de melhoria da segurança do paciente e da cultura podem ser avaliadas a partir da aplicação de instrumentos de pesquisa psicométrica que mensuram e avaliam a cultura de segurança.

Uma revisão sistemática realizada com o objetivo de identificar intervenções utilizadas para promover a cultura de segurança e avaliar sua eficácia, ressalta a melhor evidência: estratégias que compreendem múltiplos componentes, que incorporam treinamento e mecanismos para melhorar a comunicação da equipe ${ }^{(5)}$. Dessa forma, torna-se evidente a importância da realização de atividades educativas nas instituições, com o foco em melhorar a cultura de segurança, abordando principalmente a comunicação e o trabalho em equipe. O desenvolvimento de ações educativas junto aos profissionais de enfermagem é fundamental para assegurar a qualidade à saúde ${ }^{(6)}$.

O processo educativo da equipe de enfermagem proporciona o desenvolvimento de competências imprescindiveis para a segurança do paciente, como: agir, aprender, comprometer-se, assumir responsabilidades e comunicar-se ${ }^{(7)}$.

Este estudo objetiva analisar os resultados das produções científicas sobre intervenções educativas desenvolvidas com profissionais de enfermagem voltadas para melhorar a segurança do paciente e que avaliaram a cultura de segurança após essa intervenção.

\section{METODOLOGIA}

Revisão integrativa da literatura ${ }^{(8)}$, realizada de setembro/2014 a fevereiro/2015. O período de busca de cinco anos foi em virtude de encontrar o maior número possível de informações sobre o tema. Utilizou-se a pesquisa da literatura eletrônica nas bases de dados Cumulative Index to Nursing and Allied Health Literature (CINAHL), Scopus, Medical Literature Analysis and Retrieval System Online (MEDLINE), Base de Dados de Enfermagem (BDENF), Literatura LatinoAmericana e do Caribe em Ciências da Saúde (LILACS), Scientific Electronic Library Online(SciELO).

Os critérios de inclusão dos artigos foram: ser artigo de pesquisa completo, estar disponivel eletronicamente, nos idiomas português, inglês ou espanhol, no período compreendido entre 2009 até setembro de 2014, retratar intervenções educativas para melhorar a segurança do paciente e ter aplicado um instrumento de avaliação para cultura de segurança após a implementação da intervenção do estudo.

Foram elencadas as estratégias de busca: organização de descritores segundo a diferenciação entre as bases de dados; combinação de termos de busca (descritores em Ciências da Saúde-DeCS/MeSHePalavras-chave); combinação determos boolianos (and, or, not, or not). Devido às características especificas de cada base, as estratégias de busca utilizadas para localizar os artigos foram adaptadas, tendo como eixo norteador a pergunta de pesquisa e os critérios de inclusão, mantendo rigor na busca e, assim, evitando possiveis vieses.

A seleção dos estudos se deu a partir do critério de exaustividade e pertinência na coleta dos dados. Inicialmente, todo o material foi separado na forma de resumos. Posteriormente realizou-se leitura flutuante para conferência de sua validade, de acordo com o objetivo do estudo. Os que atenderam aos critérios de inclusão foram contemplados, sendo todos organizados em planilha com as principais informações: título, autores, intervenção estudada e resultados do estudo, ano e país de publicação, base de dados.

\section{RESULTADOS}

Foram encontradas 3672 publicações e, após refinamento, selecionaram-se 134 publicações. Após a leitura e análise preliminar, foram excluidas 125 referências, conforme apresentado na Tabela 1, resultando nove publicações, que configuraram a amostra desta revisão.

Tabela 1 - Categorização da seleção da amostra deste estudo. 


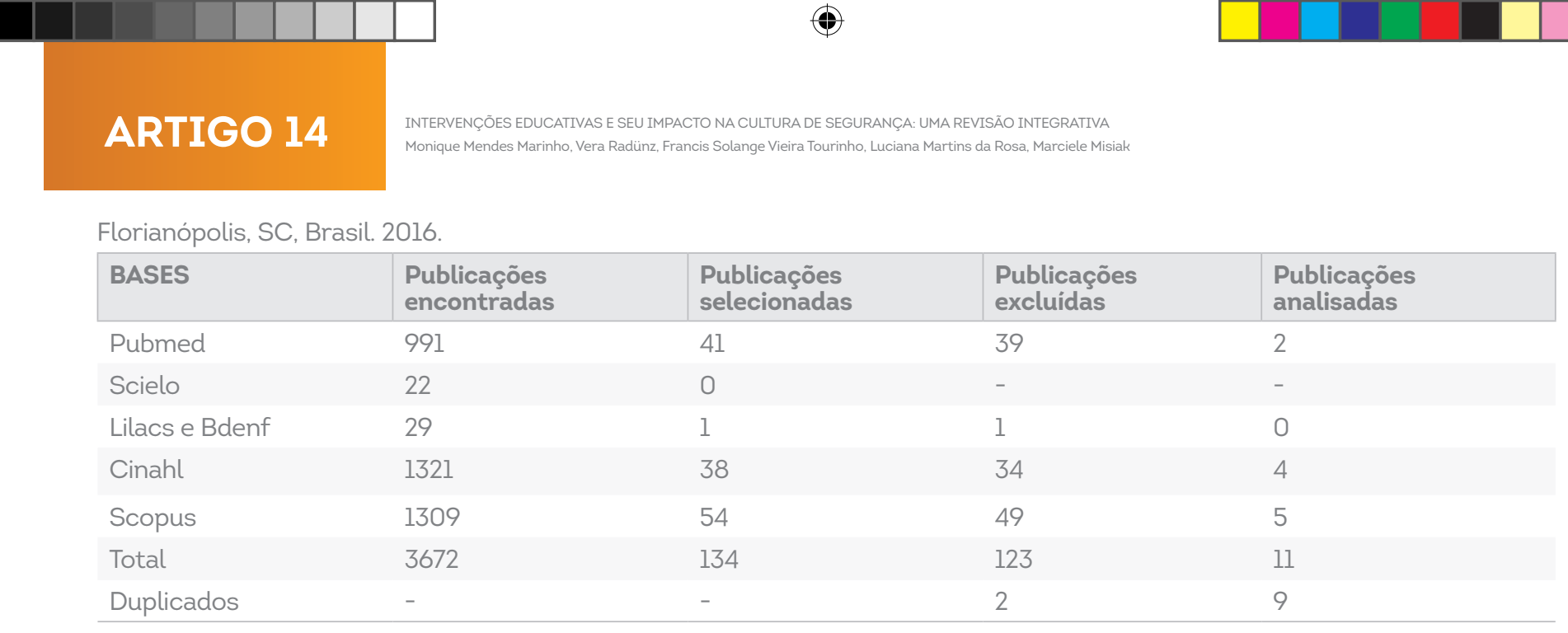

Fonte: Resultados da pesquisa.

$\mathrm{Na}$ base Scopus foram localizadas o maior número de produções, que reforça a sua magnitude na área da Enfermagem. Os resultados mostram que, em relação ao ano de publicação, 2013 teve destaque.

O maior número de publicações é proveniente dos Estados Unidos da América (EUA), com um total de seis artigos. O idioma predominante em $100 \%$ das publicações foi o inglês, denotando escassez de publicações sobre o tema nos demais idiomas selecionados para esta revisão.

O quadro 1 apresenta a síntese dos artigos selecionados nesta revisão. Em geral, os estudos utilizaram múltiplas intervenções para melhorar a segurança do paciente e avaliaram a cultura de segurança por meio do instrumento de pesquisa psicométrica SAQ (Safety Attitudes Questionnaire). A maioria dos achados obtiveram melhorias significativas nos resultados das avaliações da cultura após a intervenção.

Quadro 1 - Artigos incluidos, por base de dados, intervenção estudada, instrumento e resultados. Florianópolis, SC, Brasil. 2016.

\begin{tabular}{|c|c|c|c|c|}
\hline Titulo & Autores & Intervenção estudada & Instrumento & Resultados \\
\hline \multicolumn{5}{|c|}{ CINAHL } \\
\hline $\begin{array}{l}\text { A comprehensive obstetrics patient safety } \\
\text { program improves safety climate and culture }\end{array}$ & $\begin{array}{l}\text { Pettker, CM } \\
\text { Thung, SF } \\
\text { Raab, CA } \\
\text { Donohue, KP } \\
\text { Copel, JA } \\
\text { Lockwood, CJ } \\
\text { Funai, EF }\end{array}$ & $\begin{array}{l}\text { Programa de segurança } \\
\text { do paciente }\end{array}$ & SAO & $\begin{array}{l}\text { Aumentos consideráveis } \\
\text { em } 4 \text { dimensões da } \\
\text { avaliação da culturat }\end{array}$ \\
\hline $\begin{array}{l}\text { Didactic and Simulation Nontechnical Skills } \\
\text { Team Training to Improve Perinatal Patient } \\
\text { Outcomes in a Community Hospital }\end{array}$ & $\begin{array}{l}\text { Riley, W } \\
\text { Davis, S } \\
\text { Miller, K } \\
\text { Hansen, H } \\
\text { Sainfort, F } \\
\text { Sweet, R }\end{array}$ & $\begin{array}{l}\text { Treinamento didático } \\
\text { TeamSTEPPS e programa } \\
\text { de simulação }\end{array}$ & SAO & $\begin{array}{l}\text { Não houve diferenças } \\
\text { significativas na cultura }\end{array}$ \\
\hline $\begin{array}{l}\text { Just clean your hands: Measuring the effect } \\
\text { of a patient safety initiative on driving } \\
\text { transformational change in a health care } \\
\text { system }\end{array}$ & DiDiodato, G & $\begin{array}{l}\text { Programa educativo para } \\
\text { higienização das mãos }\end{array}$ & SAO & $\begin{array}{l}\text { Não houve mudanças } \\
\text { positivas na avaliação da } \\
\text { cultura }\end{array}$ \\
\hline
\end{tabular}

\section{CINAHL-PUBMED-SCOPUS}

The Impact of Educational Interventions on Enhancing Perceptions of Patient Safety Culture Among Jordanian Senior Nurses
AbuAlRub, RF Alhijaa, EHA
Módulos de segurança do HSPSC paciente, com 7 cursos eletrônicos
Melhora significativa em 2 dimensões

$\begin{array}{llll} & & \text { PUBMED } & \\ \text { Multiprofessional Team Simulation } & \text { O'Leary, KJ } & \text { Reuniões semanais com } & \text { SAO } \\ \text { Training, Based on an Obstetric Model, } & \text { Wayne, DB } & \text { discussões por 12 sema- } & \text { não houve melhora na } \\ \text { Can Improve Teamwork in Other } & \text { Haviley, C } & \text { navalizaram o SIDR } \\ \text { Areas of Health Care } & \text { Slade, ME } & \\ & \text { Lee, J } & \\ \text { Williams, MV } & \end{array}$




\begin{tabular}{|c|c|c|c|c|}
\hline \multicolumn{5}{|c|}{ SCOPUS } \\
\hline $\begin{array}{l}\text { Improving Teamwork: Impact of } \\
\text { Structured Interdisciplinary Rounds on a } \\
\text { Medical Teaching Unit }\end{array}$ & $\begin{array}{l}\text { O'Leary, KJ } \\
\text { Wayne, DB } \\
\text { Haviley, C } \\
\text { Slade, ME } \\
\text { Lee, J } \\
\text { Williams, MV }\end{array}$ & $\begin{array}{l}\text { Reuniões semanais com } \\
\text { discussões por } 12 \text { sema- } \\
\text { nas, utilizaram o SIDR }\end{array}$ & SAO & $\begin{array}{l}\text { Não houve melhora na } \\
\text { avaliação da cultura }\end{array}$ \\
\hline $\begin{array}{l}\text { Impact of a comprehensive patient safety } \\
\text { strategy on obstetric adverse events }\end{array}$ & $\begin{array}{l}\text { Pettker, CM } \\
\text { Thung, SF } \\
\text { Norwitz, ER } \\
\text { Buhimschi, CS } \\
\text { Raab, CA } \\
\text { Copel, JA } \\
\text { Kuczynski, E } \\
\text { Lockwood, CJ } \\
\text { Funai, EF }\end{array}$ & $\begin{array}{l}\text { Programa de segurança } \\
\text { do paciente. }\end{array}$ & SAO & $\begin{array}{l}\text { Melhora na cultura, } \\
\text { principalmente no clima } \\
\text { de trabalho em equipe }\end{array}$ \\
\hline $\begin{array}{l}\text { Interdisciplinary Collaboration to Maintain } \\
\text { a Culture of Safety in a Labor and Delivery } \\
\text { Setting }\end{array}$ & $\begin{array}{l}\text { Burke, C } \\
\text { Grobman, W } \\
\text { Miller, D }\end{array}$ & $\begin{array}{l}\text { Programa educacional } \\
\text { envolvendo discussão e } \\
\text { simulação }\end{array}$ & HSPSC & $\begin{array}{l}\text { Melhora da cultura de } \\
\text { segurança }\end{array}$ \\
\hline $\begin{array}{l}\text { A Comprehensive Patient Safety Program } \\
\text { Can Significantly Reduce Preventable Harm, } \\
\text { Associated Costs, and Hospital Mortality }\end{array}$ & $\begin{array}{l}\text { Brilli, RJ } \\
\text { McClead Jr, RE } \\
\text { Crandall, WV } \\
\text { Stoverock, L } \\
\text { Berry, JC } \\
\text { Wheeler, TA } \\
\text { Davis, JT }\end{array}$ & $\begin{array}{l}\text { Programa de segurança } \\
\text { do paciente }\end{array}$ & SAO & $\begin{array}{l}\text { Aumento significativo } \\
\text { em todo hospital nos } \\
\text { escores da cultura }\end{array}$ \\
\hline
\end{tabular}

Fonte: Resultados da pesquisa

A avaliação dos resultados das nove produções incluidas neste estudo foram agrupados em duas categorias que emergiram no intuito de atender o objetivo proposto: treinamentos didáticos e programas específicos de segurança do paciente.

$\mathrm{Na}$ categoria Treinamentos didáticos foram avaliados cinco estudos, em que são apresentados os programas de treinamentos didáticos baseados em evidências, simulações e estruturas de grupo com foco nas melhorias do trabalho em equipe, na comunicação e consequentemente na promoção de uma cultura de segurança.

Os treinamentos didáticos utilizados nos estudos selecionados foram: TeamSTEPPS (Team Strategies and Tools to Enhance Performance and Patient Safety), SIDR (Structured Inter-disciplinary Rounds), SBAR (SituationBackground-Assessment-Recommendation), Just Clean Your Hands (JCYH) e módulos de segurança do paciente do Institute for Healthcare Improvement (IHI).

$\mathrm{Na}$ categoria Programas Específicos de Segurança do Paciente foram avaliados quatro estudos, que utilizaram como intervenção a implementação de um conjunto de iniciativas voltadas para melhorar a segurança. Esses programas envolvem diversas atividades nas instituições de saúde, que iniciam com treinamento da equipe, padronização de protocolos, implementação de um sistema de notificação, fomento da comunicação, criação de sites com recomendações para a segurança, criação de um comitê, além de cursos específicos para cada área de atuação(9-10).

\section{DISCUSSÃO}

Ao analisar as produções selecionadas verificou-se que a maioria das publicações obteve melhorias estatisticamente significativas na cultura de segurança após a implementação da intervenção escolhida. Em quatro estudos perceberam redução na taxa de eventos adversos e morbidade ${ }^{(11-13,7)}$.

Em relação à categoria Treinamentos didáticos, destacase o trabalho em equipe baseado em evidências denominado TeamSTEPPS. Esse sistema tem o objetivo de aprimorar os resultados dos pacientes, a partir de melhorias na comunicação e no trabalho em equipe dos profissionais de saúde ${ }^{(14)}$. Seus princípios fundamentais são estrutura da equipe, liderança, acompanhamento da situação, apoio mútuo e comunicação(11).

A ferramenta Structured Inter-disciplinary Rounds (SIDR) foi aplicada por meio de reuniões semanais de 30-40 minutos com discussões entre os profissionais do hospital estudado. A SIDR é uma estratégia que combina um formato estruturado de comunicação e um fórum para reuniões interdisciplinares regulares, para reunir membros da equipe e melhorar a colaboração e o trabalho em equipe ${ }^{(15)}$.

O Situation-Background-Assessment-Recommendation (SBAR) é uma técnica que favorece a comunicação entre os 
profissionais sobre a condição do paciente, por meio de um mecanismo que permite uma maneira fácil e focada em definir o que será comunicado entre os membros da equipe e o que é essencial para o desenvolvimento de trabalho e promoção de uma cultura de segurança ${ }^{(16-17)}$. A técnica ocorre por sessões de formação utilizando simulação de acontecimentos reais a partir de análise de causa raiz, com discussão no final de cada sessão utilizando o SBAR ${ }^{(16)}$.

No que se refere à Análise de Causa Raiz (Root cause analysis - RCA), o impacto do seu uso periódico na área de saúde possibilita facilitar a aprendizagem organizacional como uma estratégia de melhoria. Os erros, quase-erros e eventos notificados são analisados a partir da RCA, permitindo aprender com os próprios erros, dificultando uma nova ocorrência.

O Just Clean Your Hands (JCYH) é um programa de educação e formação baseada em evidências para as melhores práticas de higienização das mãos para os profissionais de saúde. Desenvolvido pelo governo de Ontário, Canadá, como uma iniciativa para melhorar a segurança do paciente. Sugere-se a avaliação da cultura de segurança, porém sem nenhum instrumento padrão ouro para tal(18-19).

Os módulos de segurança do paciente do Institute for Healthcare Improvement (IHI) fazem parte de um programa chamado Programa IHI Open School, com o objetivo de contribuir para a formação e aquisição de competências dos profissionais para melhoria da qualidade e da segurança do paciente ${ }^{(20)}$. Os sete cursos eletrônicos utilizados no estudo ${ }^{(12)}$ abordam contextos essenciais para segurança, apresentados de forma verbal, com incentivo para participação ativa nos cursos $^{(20)}$.

O modelo de simulação realística, utilizado como treinamento em um estudo, foi baseado no evento clínico real gravado que serviu de cenário de simulação. Foi projetado por uma equipe multiprofissional, facilitando a aplicação de habilidades clínicas relevantes. Após cada simulação, ocorreu discussão sobre o desempenho da equipe, críticas e opiniões. Esse modelo proporcionou uma oportunidade de comunicação, cooperação e confiança na equipe ${ }^{(21)}$.

As estratégias de treinamento de equipe apresentadas demonstram técnicas específicas que podem ser adaptadas para variados contextos de serviços de saúde, mesmo em situações onde há poucos recursos disponíveis, em situações de restrições financeiras.

A categoria Programas Especificos de Segurança do Paciente discute os resultados de quatro estudos, que utilizaram um conjunto de iniciativas para melhorar a segurança. Um estudo utilizou um programa que reforça quanto ao uso de um logotipo criado para sinalizar e transmitir uma mensagem consistente ${ }^{(13)}$. Além disso, a realização de um treinamento básico de prevenção de erro para todos os 8 mil funcionários clínicos e não clínicos, e 600 líderes que receberam treinamento em métodos de liderança. 0 treinamento realizado é diferencial nesse programa ${ }^{(13)}$, pois treinou profissionais de saúde e de outras áreas e líderes, reforçando o envolvimento de todos que atuam de forma direta ou indireta no cuidado.

O programa de segurança do paciente deve ser uma realidade de todas as instituições de saúde, pois reúne diversas atividades para melhorar a qualidade e a segurança dos serviços. Existem iniciativas de baixo custo e complexidade que são relevantes, como padronização de protocolos, sistema de notificação e incentivo à cultura de segurança.

Um hospital envolveu 372 profissionais de saúde no programa educacional, envolvendo discussão e simulação. O programa apresenta protocolos para a segurança do paciente, garantem um ambiente livre de culpa e proporcionam uma oportunidade de melhorar a comunicação entre a equipe. Além disso, obteve melhoria na avaliação da cultura após a implementação do programa(21).

A iniciativa de melhorar a segurança do paciente principalmente por meio de um programa específico é um esforço relevante que refletirá em resultados aos pacientes. Serve como referência para a instituição, pois reconhece a necessidade de contribuir para um cuidado seguro, protegendo os profissionais do sentimento de culpa e incompetência, e, aos pacientes, de perigos e danos que podem ser evitados.

Em relação ao objetivo desta revisão, observou-se a identificação das diversas atividades educativas que poderiam ser replicadas em outras realidades, podendo combinar atividades de diferentes estudos, com adaptações para as 
características locais e posteriormente avaliar o impacto na cultura de segurança.

$\mathrm{O}$ ato de planejar e implementar qualquer iniciativa para melhorar a segurança é um passo importante para diminuir os inúmeros danos causados por uma prática insegura. Destacase que os efeitos dessas intervenções podem demorar a serem enraizados na cultura local, pois foram muitos anos cultivando uma cultura de culpa, castigo e silêncio que podem comprometer os esforços para mudar esse cenário.

Como limitação desta revisão, destaca-se o reduzido número de produções selecionados neste estudo. Inexistem estudos brasileiros, dificultando a avaliação sobre um método considerado adequado para avanços na segurança do paciente.

\section{CONSIDERAÇÕES FINAIS}

A implementação bem sucedida de atividades educativas está atrelada ao desejo da gestão de mudança organizacional, permitindo espaço na instituição e apoio junto aos profissionais de saúde, principalmente os de enfermagem, que dispõe de uma carga de trabalho aumentada.

As mudanças na cultura de segurança podem não ser demonstradas nas avaliações psicométricas, porém os resultados impactam na redução na taxa de eventos adversos, morbidade e mortalidade. Quanto à escolha da intervenção para melhorar a cultura de segurança, destacam-se os diversos treinamentos didáticos da equipe e a implementação de programas de segurança nos serviços, que, na grande maioria, contribuem para melhores escores da avaliação da cultura.

Ressalta-se que todos os estudos analisados são de procedência internacional, portanto, contextualizam realidades e características diferentes da nossa. Dessa forma, faz-se necessário o desenvolvimento de estudos que identifiquem o impacto de atividades educativas na avaliação da cultura na realidade brasileira.

\section{REFERÊNCIAS}

1. World Health Organization (WHO).Geneva: WHO. Alliance for Patient Safety: the conceptual framework for the international classification for patient safety. 2009 [acesso em 08 fev 2015]. World. Disponivel: < http:// www.who.int/patientsafety/taxonomy/icps_full_report.pdf>

2. World Health Organization. Patient safety: global priorities for research in patient safety. 2008

3. Kohn KT, Corrigan JM, Donaldson MS editors. To err is human: building a safer health system. [internet] Washington: National Academy Press; 2000. [acesso em 27 maio 2013 ] Disponivel: <http://www.nap.edu/openbook. php?isbn $=0309068371$

4. Salas E, Diazgranados D, Weaver SJ, King H. Does team training work? Principles for health care. Acad Emerg Med. 2008:15:1002-9.

5. Weaver SJ, Lubomksi LH, Wilson RF, Pfoh ER, Martinez KA, Dy SM. Promoting a Culture of Safety as a Patient Safety Strategy: A Systematic Review. Ann Intern Med.2013:158, 369-374.

6. Mira VL, Peduzzi M, Melleiro MM, Tronchin DMR, Prado MFF, Santos PT, et al. Análise do processo de avaliação da aprendizagem de açốes educativas de profissionais de enfermagem. Rev. esc. enferm. USP. 2011 [acesso em 29 jan 2015 ]45, n.esp. Dec.

7. Kobayashi RM, Leite MMJ. Desenvolvendo competências profissionais dos enfermeiros em serviço. Rev. bras. enferm. 2010 [acesso em 21 apr 2015];63(2)243-249.

8. Soares CB, Hoga LAK, Peduzzi M, Sangaleti C. Yonekura T, Silva DRAD. Revisão integrativa: conceitos e métodos utilizados na enfermagem. Rev Esc Enferm USP. 2014; 48(2):335-45.

Pettker CM, Thung SF, Norwitz ER, Buhimschi CS, Raab CA, Copel JA, et. al. Impact of a comprehensive patient safety strategy on obstetric adverse events. Am J Obstet Gynecol. 2009; 492:1-8.

9. Pettker CM, Thung SF, Raab CA,Donohue KP, Copel JA, Lockwood CJ, et. al. A comprehensive obstetrics patient safety program improves safety climate and culture. Am J Obstet Gynecol. 2011; 216:1-6.

10. Riley W, Davis S, Miller K, Hansen H, Sainfort F, Sweet R. Didactic and simulation nontechnical skills team training to improve perinatal patient outcomes in a community hospital. Jt Comm J Qual Patient Saf. 2011; $37(8): 357-64$
11. Abualrub RF, Abualhijaa EH. The impact of educational interventions on enhancing perceptions of patient safety culture among Jordanian senior nurses. Nurse Forum.2014:49(2):139-50.

12. Brilli RJ, McClead REJr, Crandall WV, Stoverock L, Berry JC, Wheeler TA et al. A comprehensive patient safety program can significantly reduce preventable harm, associated costs, and hospital mortality. J Pediatr. 2013:163(6):1638-45.

13. Teamstepps National Implementation [Internet]. [acesso 31 jan 2015] About TeamSTEPPS. Disponivel: http://www.teamsteppsportal.org/.

14. O'Leary KJ, Wayne DB. Haviley C. Slade ME, Lee J, Williams MV. Improving teamwork: impact of structured interdisciplinary rounds on a medical teaching unit. J Gen Intern Med. 2010:25(8):826-832.

15. Van der Nelson HA, Siassakos D, Bennett J, Godfrey M, Spray L, Draycott T. et. al. Multiprofessional team simulation training, based on an obstetric model, can improve teamwork in other areas of health care. Am J Med Qual. 2014:29(1):78-82.

16. Institute for Healthcare Improvement. IHI; 2015 [acesso Ol fev 2015] SBAR Technique for Communication: A Situational Briefing Model. Disponivel.http://www.hi.org/resources/Pages/Tools/ SBARTechniqueforCommunicationAsituationalBriefingModel.aspx.

17. Didiodato, G. Just clean your hands: Measuring the effect of a patient safety initiative on driving transformational change in a health care system. American Journal of Infection Control. 2013:1-3.

18. Ontario Ministry Of Health. [acesso 31 jan 2015] Just Clean Your Hands for health care professionals. Disponivel: <http://www.publichealthontario. ca/en/BrowseByTopic/InfectiousDiseases/JustCleanYourHands/Pages/ JCYH-background.aspx\#.VM6wktLF98E

19. Institute for Healthcare Improvement. IHI; 2015 [acesso 21 mar 2015] Open School. Disponivel: http://app.ihi.org/lms/onlinelearning. aspx?\&CatalogGuid=a5d865d5-9c8d-4db8-977f-8d8600cba5bf.

20. Burke C, Grobman W, Miller D. Interdisciplinary collaboration to maintain a culture of safety in a labor and delivery setting. J Perinat Neionatal Nurs. 2013:27(2):113-123. 